\title{
Continuous Development and Cultivation of Soybean as A Result of Multigamma Irradiation through Final Purifying Method
}

\author{
Bartholomeus Pasangka1 ${ }^{*}$, Irvandi Gorby. P2 \\ ${ }^{1}$ Department of Physical and Agricultural Sciences, Nusa Cendana University Indonesia \\ ${ }^{2}$ Department of Mathematics Faculty of Sciences and Engineering Nusa Cendana University Indonesia.
}

DOI: $10.29303 /$ jppipa.v7i2.611

\section{Article Info}

Received : January 29th, 2021

Revised : March 29th, 2021

Accepted: April 3th, 2021

\begin{abstract}
Soybean was the first legumes in Indonesia with production more decreased, while fulfill of public societies were increased. In order that, it was necessary developed and cultivated with technology application. The main problem investigated in this work was the continuous development of Soybean as a result of multi-gamma irradiation on the first year of research. On the second, third, and the fourth year of research, varieties of mutants were purified by carefully selection while growth of plant until to harvest. The selection type was individual selections. In the same time was done multi-locations test. This work obtained five variations of superior varieties of mutant after purifying, which production range about $3.58-5.12 \mathrm{t} / \mathrm{ha}$, and the total average production about $4.48 \mathrm{t} / \mathrm{ha}$. Range production of initial variety about $1.96-2.61 \mathrm{t} / \mathrm{ha}$ and average production of initial (control) soybean about $2.40 \mathrm{t} / \mathrm{ha}$. Percentage rate production of superior selected mutant variety after purifying about $46.47 \%$.
\end{abstract}

Keywords: Development; Cultivation, Soybean; Multigamma; Irradiation; Purifying.

Citation: Pasangka, B., \& Gorby, I. (2021). Continuous Development and Cultivation of Soybean as A Result of Multigamma Irradiation Through Final Purifying Method. Jurnal Penelitian Pendidikan IPA, 7(2), 156-166. doi:https://doi.org/10.29303/jppipa.v7i2.611

\section{Introduction}

Soybean called Glycine $\max L$ was the first important legumes in Indonesia, which high content of protein, low cholesterol, and it price was cheap (Irwan, 2006; Mansyuriadi, 2015). On every year, requirement of soybean in National scale always to be increased (Statistics Center Organization, 2014; Zakaria et al. 2018), while its production decreased. The production of soybean on 2004 in the country only $1,878,898$ tons, while requirement of soybean in National scale on this time achieve 2,955,000 tons (Indrawan, 2009; Hasan et al., 2015). On the same time, the average production of soybean in the world achieves 1.9 tons per hectare, and on the National scale the average production of soybean achieves $1.2 \mathrm{t} /$ ha (Atman, 2014). The consequence of decreasing of soybean production in the last time was the government has to import the soybean on every year as many as 300 thousand tons (Arsyad and Syam, 2004;
Azis, 2014; Aimon et al., 2014). The request projection of soybean as the fasting 2018 achieve 6.11 million tons, while the production of soybean in 2003 was only 672,000 tons and the production of soybean in 1992 achieve 1.87 million tons (Hilman et al., 2004; Atman, 2006).

The low of commodity production of soybean per hectare in Indonesia influenced by several factors, among others the using of superior seeds, possess a dry soil, germ (Baliardi and Saleh, 1989; Marwoto et al., 2017.), climate, poor of micro-elements, growing hormone, and soil factor (McFarlane, I. and O'Connor, 2014). The production of soybean in the level of the farmer in Indonesia until now is very low, therefore it was necessary developed and cultivated by using the application of modern technology like as irradiation. Soybean was one important staple supply in industry of tofu, tempeh, milk of soybean, cakes, etc (Saktyanu, 2014; Nancy et al., 2018). 
In the first year of the research has been obtained five varieties of mutant with using multi-gamma irradiation, which continuous developed and cultivated in the four years (the second, the third, and the fourth year of research). Dosage standard of multi-gamma irradiation used for breeding of soybean in the first research was 3,500 radiations (Darussalam, 1989). The dosage was decided by Darusalam that is mutation of seeds plant: 1,000 radiations up to 4,000 radiations. The seeds of soybean were irradiated while 30 minutes in the chamber of multi-gamma sources.

The research of the second, third, and fourth years was focused in purifying on every varieties of mutant selected to obtain homogeneous plant on each variety of mutant which have superior characteristics. The general characteristics of superior selected mutant of soybean varieties obtained on the final purifying were: high production, adapted to possess a dry soil, and tolerant to germ (Krisnawati et al., 2015; Okada, et al., 1988), the age of mutant was shorter than initial, tolerant to germ specially viruses (Azzamy, 2015), the quality of seed increase (content of protein and fat) (Irwan, 2006).

This research aimed: to continuously develop and cultivate of selected mutant varieties of local soybean through purifying while three years for obtaining several superior selected varieties through purifying, multi-location test, and carefully selection.

\section{Method}

\section{Material Study}

The important equipment used in this work (purifying and multi-location test level) were tractors, protein analyzer, water content analysis, digital balance, the huller of dry soybean fruit, and other equipment. The sample for purifying was mutant varieties of soybean seeds found on the first research, and initial sample of soybean as a comparison.

\section{Description of Area}

On the first and second purifying of selected mutant varieties in this research located at East Nusa Tenggara. The third purifying (final purifying) located in four provinces that are province of East Nusa Tenggara (altitude: 800 meters from sea level), South Sulawesi (altitude: $2050 \mathrm{~m}$ from sea level), West Sulawesi (altitude: 850 meters from sea level), and Middle Sulawesi (altitude: 1650 meters from sea level). This case proposed for testing of multi-locations after the first and second purifying. The all places used in province of East Nusa Tenggara have the same states among other illumination, the structure of soil, possess a dry soil, salt and lime levels. The average of illumination of this location was 5 hours every day. The area conditions used in the four provinces were different, so, can be tested adaptation characteristics of mutant soybean after purifying to different cases of planting area. Range of temperature in the fourth provinces at the planting locations about $16^{\circ} \mathrm{C}$ up to $20^{\circ} \mathrm{C}$.

The methods used in this research comprises of observation, sampling, carefully selection, testing, purifying, comparison, and interpretation. Data collection and analysis were done with observation, measuring, calculations, analyzing of protein and water content on treatment and control samples. The all physical and chemical characteristics on treatment and control samples ware compared for quality control.

The several steps of research procedures comprised of: 1) To prepare and cultivate land for planting of soybean and corn seeds. 2) To soak planting area. 3) To plant seeds of soybean with intercropping model after two days soaking. 4) To observe growth of soybean seeds on 7 days after planting appropriated to random samples for calculating growth percentage. 5) To do watering if it is necessary (appropriated to weather condition). 6) To weed and fertilize plant for obtaining maximum production. 7) To observe tenacity of germ, growth in dry area, and others physical and chemical characteristics which were needed for comparison standard like as growth time, flowered age, calculating of growth percentages, measuring of mass per 1,000 seeds of soybean, determination production ranges, calculating of average production, and plants selecting. On harvest resemble, carefully selection is done, measuring of high plant, and measuring of mass per 1,000m seeds after harvest. 8) To analyze of protein content. 9) To dry and final carefully select of superior varieties of seeds. Drying condition take time on $06.00 \mathrm{a}$. $\mathrm{m}$ up to 09.00 a.m. 10) To compare physical and chemical characteristics between control (initial variety of soybean) and treatment samples (superior mutant varieties). 11) The final procedure is to put enough insecticide sufficient on superior seeds varieties, so can be freed from germ, and storage in plastics pocket for continuously development and cultivation. Note that the first selection of soybean is done since plant age about one month, the second selection during two months, $\mathrm{M}_{\mathrm{on}}$ selection since near to harvest, and third (final) selection after harvest.

\section{Observe and Measure Variables}

Amount of physical and chemical characteristics of soybean on control and treatment samples during growth and after harvest were observed and measured consist of adaptation, tenacity of germ, grow time, flowered age, plant high, mass per 1,000 seeds, protein content, production ranges, average production, and increasing percentage of production. 


\section{Research Design}

There are two groups chosen in research that are control (initial variety) and treatment samples (superior selected mutant varieties). The treatment samples carefully selected from mutant as a result of multigamma irradiation on first research. The all samples planted in the same time and different location (multilocation test). Research design clearly shown on Figure 1.

\section{Statistical Formulation for Data Analysis}

There are several variables calculated by statistical formulation like as growth percentage, average production, total average production, and increasing percentage of production. For calculating of growth percentage, control and treatment samples randomly chosen as many as six groups. On every group is taken number of sample test about 100 seeds. Number of sample seeds is not growth observed and calculated on every group.

Growth percentage calculated by statistical formulation (Pasangka and Refli, 2013b., Pasangka and Refli, 2016., Pasangka, 2019).

$$
G P=\left(\frac{T_{A S}-A_{S G}}{T_{A S}}\right) \times 100 \%
$$

where $G P$ is growth percentage (\%), $T_{A S}$ is the number of seeds total which planted, $A_{S G}$ is the number of seeds was not growth.

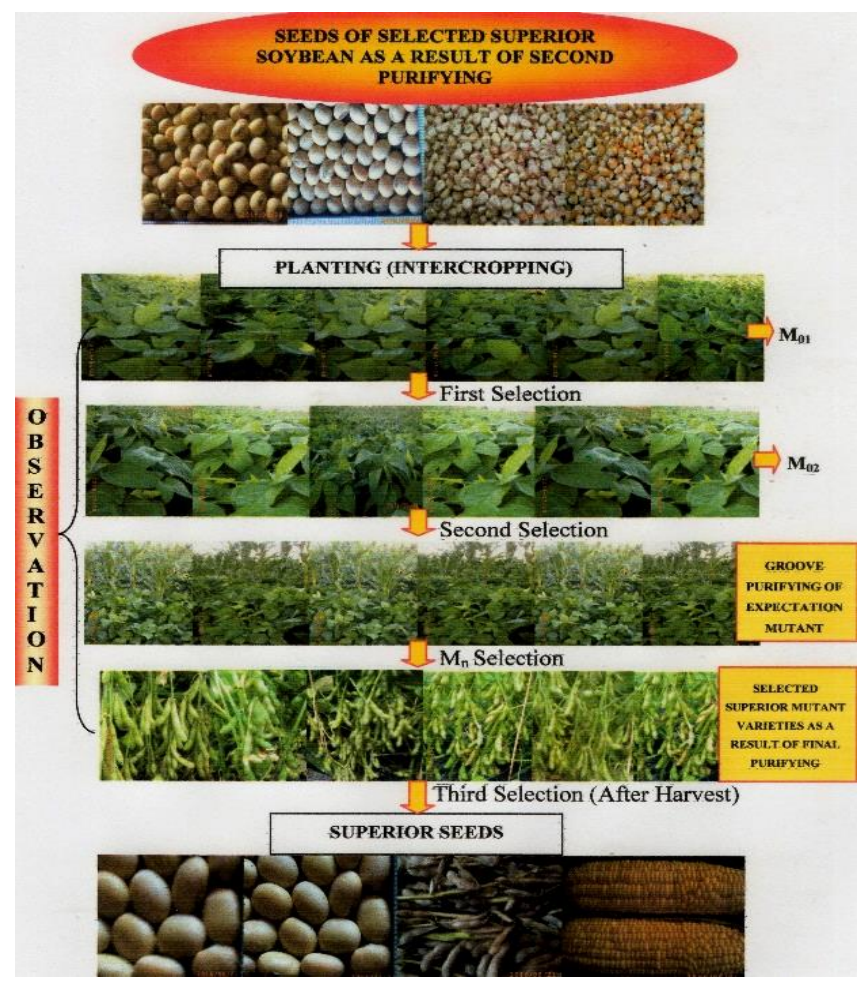

Figure 1. Research design
Equation (2) up to Equation (6) calculated average production of treatment samples on every selected mutant variety and control sample at four provinces and six planting locations (Pasangka and Refli, 2013b., Pasangka and Refli, 2016., Pasangka, 2019).

Mutant-1 Variety $\left(M_{1} V\right)$ :

$A_{P T S M(1)}=\frac{P_{L 1}+P_{L 2}+P_{L 3}+P_{L 4}+P_{L 5}+P_{L 6}}{6}$

where: $A_{P T S M(1)}$ is average production of treatment sample of mutant-1 variety at six planting location, $P_{L i}, P_{L 2}, P_{L 3}, P_{L 4}, P_{L 5}, P_{L 6}$ are mutant (treatment) and control production, and $n=1,2,3,4,5,6$ (planting location number).

Mutant -2 Variety $\left(M_{2} V\right)$ :

$A_{P T S M(2)}=\frac{P_{L 1}+P_{L 2}+P_{L 3}+P_{L 4}+P_{L 5}+P_{L 6}}{6}$

where: $A_{P T S M(2)}$ is average production of treatment sample of mutant-2 variety

Mutant-3 variety

$A_{P T S M(3)}=\frac{P_{L 1}+P_{L 2}+P_{L 3}+P_{L 4}+P_{L 5}+P_{L 6}}{6}$

where: $A_{P T S M(3)}$ is average production of treatment sample of mutant-3 variety at six planting locations.

Mutant-4 variety

$A_{P T S M(4)}=\frac{P_{L 1}+P_{L 2}+P_{L 3}+P_{L 4}+P_{L 5}+P_{L 6}}{6}$

$\left(M_{4} V\right)$ :

where: $A_{P T S M(4)}$ is average production of treatment sample of mutant- 4 variety at six planting locations.

Mutant-5 variety

$A_{P T S M(5)}=\frac{P_{L 1}+P_{L 2}+P_{L 3}+P_{L 4}+P_{L 5}+P_{L 6}}{6}$

$\left(M_{5} V\right)$ :

where: $A_{P T S M(5)}$ is average production of treatment sample of mutant- 5 variety at six planting locations.

Initial variety:

$A_{P C I V}=\frac{P_{L 1}+P_{L 2}+P_{L 3}+P_{L 4}+P_{L 5}+P_{L 6}}{6}$

where: $A_{P C I V}$ is average production of control sample (initial variety) at six planting locations.

For calculation of total average production of superior mutant variety used statistical formulation (Pasangka and Refli, 2013b., Pasangka and Refli, 2016., Pasangka, 2018).

$A_{P T S M(t)}=\frac{A_{P T S M(1)}+A_{P T S M(2)}+A_{P T S M(3)}+A_{P T S M(4)}+A_{P T S M(5)}}{5}$

where: $A_{P T S M(t)}$ is total average production of selected superior mutant variety on final purifying at four provinces and five variations of superior selected mutant varieties.

Statistical formulations calculate increasing percentage of production on every selected superior mutant variety use Equation (9) up to Equation (13) 
(Pasangka and Refli, 2013., Pasangka and Refli, 2016., Pasangka, 2019):

Mutant-1 variety $\left(M_{1} V\right)$ :

$$
I_{P P S S M(1)}=\left(\frac{A_{P T S M(1)}-A_{P C I V}}{A_{P T S M(1)}}\right) \times 100 \%
$$

where: $I_{P P P V M(1)}$ is increasing percentage of production of selected superior mutant- 1 variety at four provinces on final purifying.

Mutant-2 variety $\left(\left(M_{2} V\right)\right.$ :

$$
I_{P P S M(2)}=\left(\frac{A_{P T S M(2)}-A_{P C I V}}{A_{P T S M(2)}}\right) \times 100 \%
$$

where: $I_{P P S S M(2)}$ is increasing percentage of production of selected superior mutant-2 variety at four provinces on final purifying.

Mutant-3 variety $\left(M_{3} V\right)$ :

$$
I_{P P S S M(3)}=\left(\frac{A_{P T S M(3)}-A_{P C I V}}{A_{P T S M(3)}}\right) \times 100 \%
$$

where: $I_{P P S S(3)}$ is increasing percentage of production of selected superior mutant-3 variety at four provinces on final purifying.

Mutant-4 variety $\left(M_{4} V\right)$ :

$$
I_{P P S S M(4)}=\left(\frac{A_{P T S M(4)}-A_{P C I V}}{A_{P T S M(4)}}\right) \times 100 \%
$$

where: $I_{P P S S(4)}$ is increasing percentage of production of selected superior mutant- 4 variety at four provinces on final purifying.

Mutant-5 variety $\left(M_{5} V\right)$ :

$$
I_{P P S S M(5)}=\left(\frac{A_{P T S M(5)}-A_{P C I V}}{A_{P T S M(5)}}\right) \times 100 \%
$$
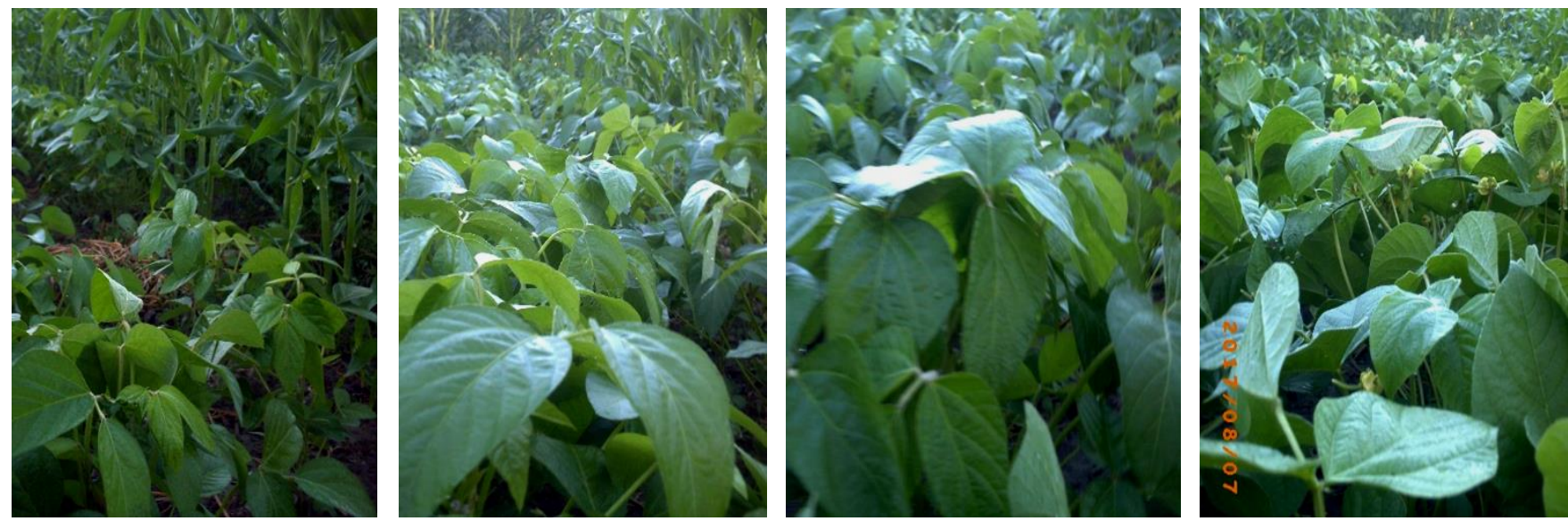

Figure 2. Mutant-1 variety $\left(\mathrm{M}_{1} \mathrm{~V}\right)$ Figure 3. Mutant-2 variety $\left(\mathrm{M}_{2} \mathrm{~V}\right)$

Figure 4 . Mutant-3 variety $\left(\mathrm{M}_{3} \mathrm{~V}\right)$

Figure 5. Mutant-4 variety $\left(\mathrm{M}_{4} \mathrm{~V}\right)$
Where: $I_{P P S S M(5)}$ is increasing percentage of production of selected superior mutant- 5 variety at four provinces on final purifying.

Statistical formulation calculates average percentage total of production of selected mutant variety at four provinces (six planting locations) on final purifying of soybean is.

$A_{\text {PTPSWV }}=\left(\frac{I_{\text {PPSSM (1) }}+I_{\text {PPSSM (2) }}+I_{\text {PPSSM (3) }}+I_{\text {PPSSM(4) }}+I_{\text {PPSSM(5) }}}{5}\right)$

where: $A_{P T P S M V}$ is average percentage total of production of selected mutant variety at four provinces on final purifying.

\section{Result and Discussion}

Results of Observation, Measurement, and Calculation Growth types of selected superior mutant varieties on final purifying at four provinces (six planting area) respectively shown on Figure 2 up to Figure 6. That Figures also show five examples of variations of selected superior mutant varieties on final purifying. Figure 7 up to Figure 11 show fruits of five variations of selected superior mutant varieties on final purifying at four provinces. Figure12 up to Figure 16 show seeds of five variations of selected superior mutant varieties on final purifying at four provinces, and Figure 17 shows one example of seeds of initial variety. Figure 18 show one example of color corn as a intercropping (is not investigated in this work) of soybean plant, and it is also as a result of multi-gamma irradiation on research in 2009 (Pasangka and Jaelani, 2010) 


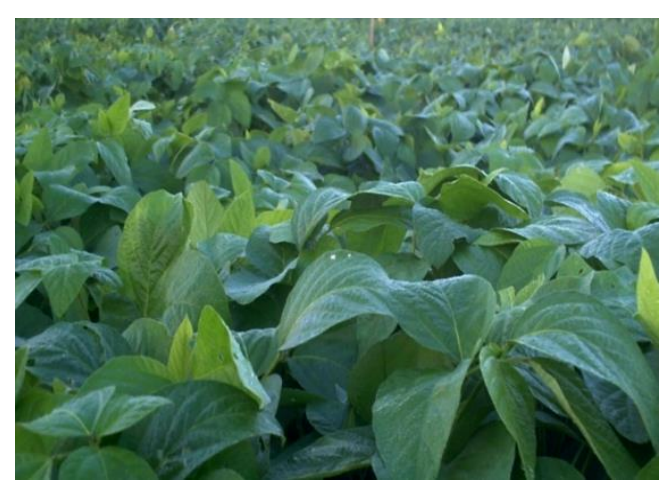

Figure 6. Mutant-5 variety $\left(\mathrm{M}_{5} \mathrm{~V}\right)$

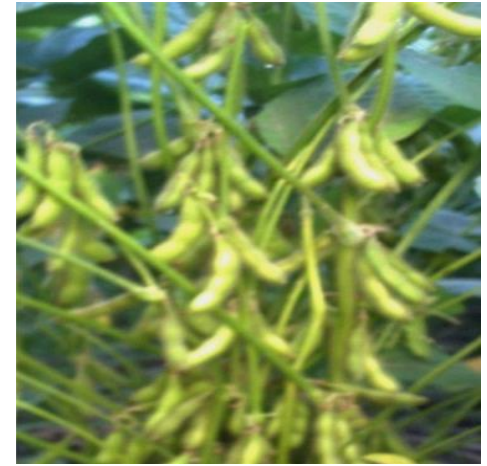

Figure 7. $\mathrm{M}_{1} \mathrm{~V}$

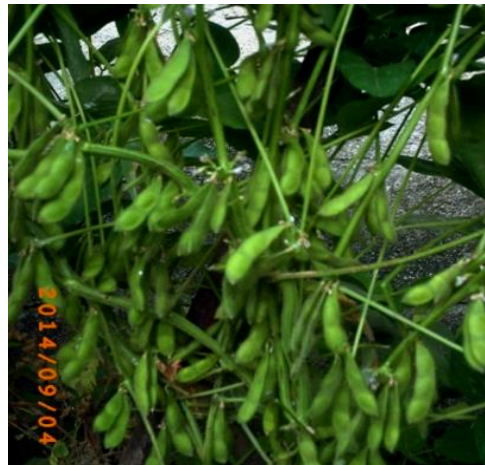

Figure $8 . \mathrm{M}_{2} \mathrm{~V}$

Figure 2 up to Figure 6 show growth of five variations of superior mutant varieties of soybean on final purifying at four provinces on age 37 days after planting.

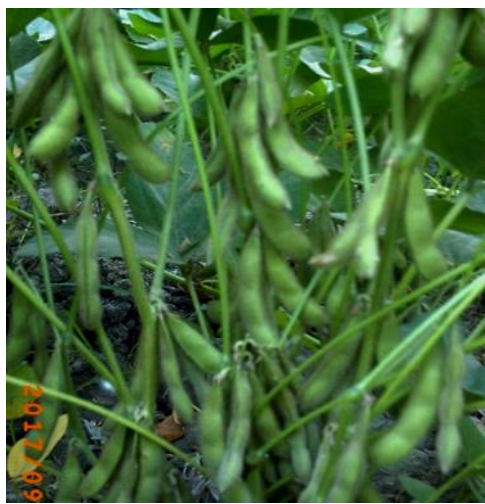

Figure $9 . \mathrm{M}_{3} \mathrm{~V}$

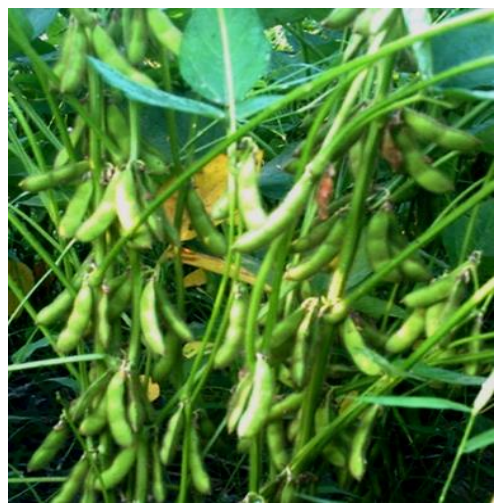

Figure-10. $\mathrm{M}_{4} \mathrm{~V}$

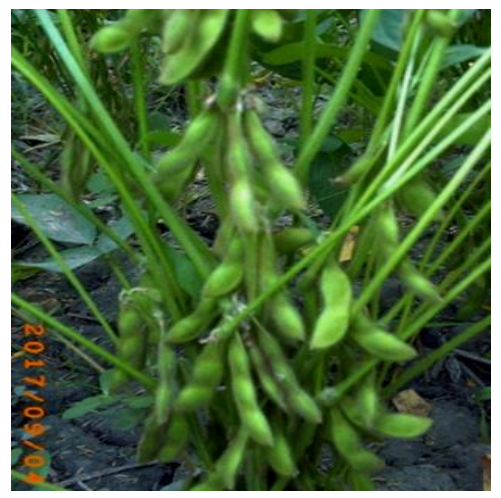

Figure $11 . \mathrm{M}_{5} \mathrm{~V}$

Figure 7 up to Figure 11 show fruits of five variations of superior varieties of soybean as a result of multi-gamma irradiation on final purifying on age about 54 days after planting.

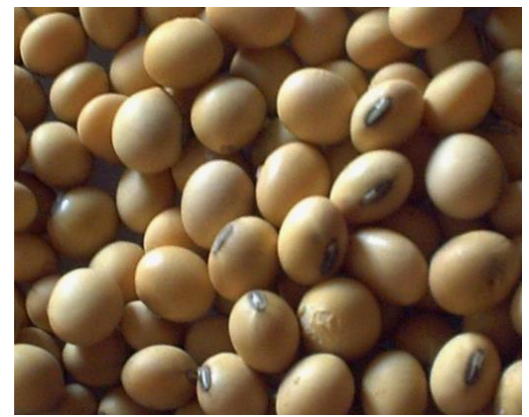

Figure 12. $\mathrm{M}_{1} \mathrm{~V}$

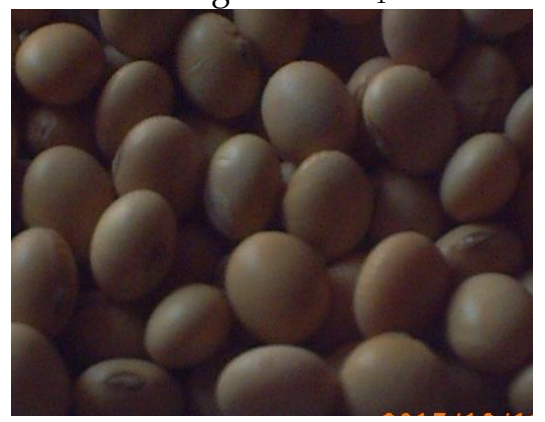

Figure 15. $\mathrm{M}_{4} \mathrm{~V}$

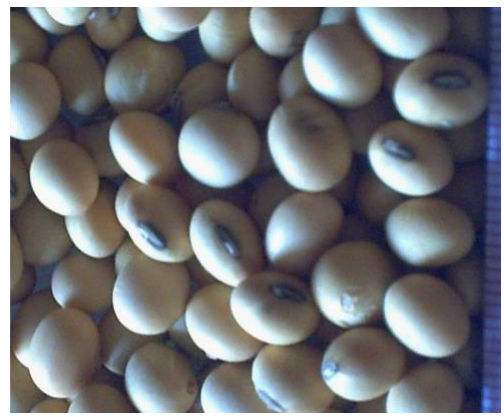

Figure $13 . \mathrm{M}_{2} \mathrm{~V}$

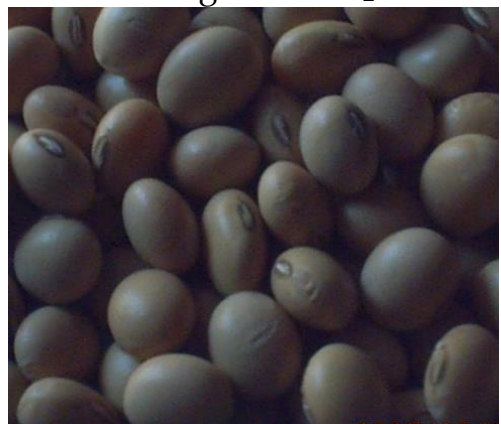

Figure $16 . \mathrm{M}_{5} \mathrm{~V}$

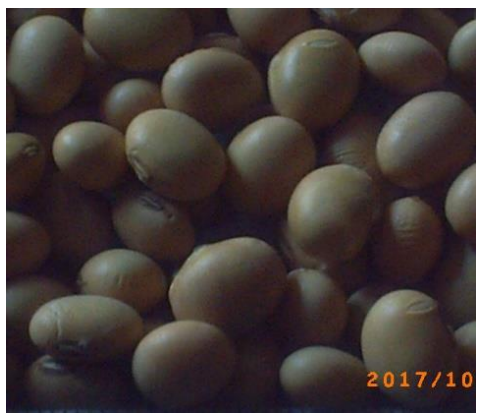

Figure $14 . \mathrm{M}_{3} \mathrm{~V}$

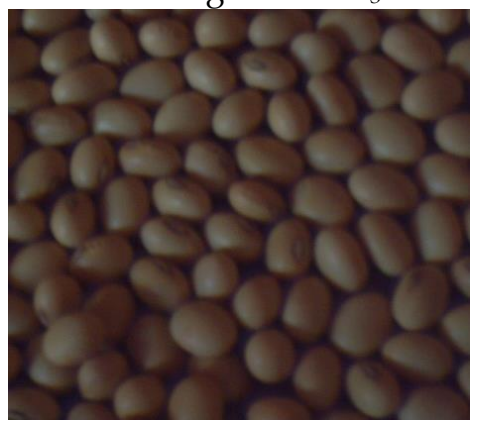

Figure 17. Initial variety

Figure 12 up to Figure 16 show seeds of five variations of superior varieties of soybean as a result of multi-gamma irradiation on final purifying. 


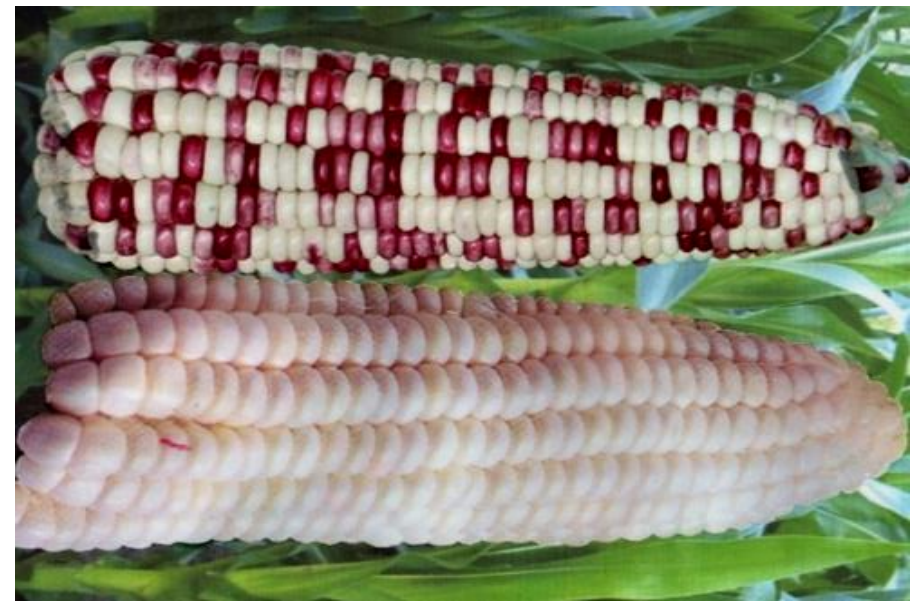

Figure 18 shows one example of color purplish-white sweet corn as a result of multi-gamma irradiation on research in 2009. (Corn in this research is not investigated, only as an intercropping plant for shelter or protection of Vigna Radiata L plants).

Results of observation on pre growth of seeds on treatment and control samples for calculating growth percentage, several physical and chemical characteristics were observed, measured, and calculated, and also production level at four provinces on final purifying prepared respectively in Table 1, Table 2, and Table 3.

Table 1. The number of seeds is not grown on control and treatment samples on final research (final purifying) at six locations in four provinces.

\begin{tabular}{|c|c|c|c|c|c|c|c|}
\hline \multirow{2}{*}{ No } & & \multicolumn{5}{|c|}{ Mutant Varieties } & \multirow{2}{*}{$\begin{array}{l}\text { Initial Variety } \\
\text { IV }\end{array}$} \\
\hline & & $\mathrm{M}_{1} \mathrm{~V}$ & $\mathrm{M}_{2} \mathrm{~V}$ & $\mathrm{M}_{3} \mathrm{~V}$ & $\mathrm{M}_{4} \mathrm{~V}$ & $\mathrm{M}_{5} \mathrm{~V}$ & \\
\hline \multicolumn{8}{|c|}{ 1) Fukdale province of East Nusa Tenggara } \\
\hline & & 1 & 2 & 4 & 2 & 6 & 9 \\
\hline \multirow[t]{2}{*}{ 2) } & Oesao Province & usa Tenggara & & & & & \\
\hline & & 2 & 4 & 4 & 4 & 6 & 8 \\
\hline \multirow[t]{2}{*}{ 3) } & Tinoring provin & h Sulawesi & & & & & \\
\hline & & 3 & 5 & 4 & 5 & 7 & 10 \\
\hline 4) & Padang provinc & $\begin{array}{l}\text { Sulawesi } \\
2\end{array}$ & 3 & 6 & 3 & 5 & 12 \\
\hline \multirow[t]{2}{*}{ 5) } & Mamuju provin & Sulawesi & & & & & \\
\hline & & 3 & 5 & 4 & 3 & 8 & 14 \\
\hline \multirow[t]{2}{*}{ 6) } & Tentena provinc & le Sulawesi & & & & & \\
\hline & & 4 & 5 & 5 & 4 & 7 & 16 \\
\hline & rage & 2.5 & 4.0 & 4.5 & 3.5 & 6.5 & 11.5 \\
\hline & wth percentage & $95 \%$ & $92 \%$ & $91 \%$ & $93 \%$ & $87 \%$ & $77 \%$ \\
\hline \multicolumn{5}{|c|}{ Average of growth percentage of selected superior mutant varieties } & $91.60 \%$ & & \\
\hline
\end{tabular}

Table 2. Physical and chemical characteristics were observed, measured, and calculated of control and treatment samples (selected superior mutant varieties) on final purifying (Pasaangka and Refli, 2013a).

\begin{tabular}{|c|c|c|c|c|c|c|c|}
\hline \multirow{2}{*}{ No } & \multirow{2}{*}{ Description } & \multirow{2}{*}{$\begin{array}{l}\text { Control } \\
\text { Sample }\end{array}$} & \multicolumn{5}{|c|}{ Selected Superior Mutant Varieties $\left(M_{n} V\right), n=1,2,3,4,5$} \\
\hline & & & 1 & 2 & 3 & 4 & 5 \\
\hline 1 & Time growth on every variety $(\mathrm{d}$ a $\mathrm{p}$ ) & 8 & 5 & 5 & 3 & 3 & 4 \\
\hline 2 & $\begin{array}{l}\text { Average of time growth on superior } \\
\text { selected mutant varieties (d. a. p) }\end{array}$ & & & & 4 & & \\
\hline 3 & Growth percentage (\%) & 77.00 & 95.00 & 92.00 & 91.00 & 93.00 & 87.00 \\
\hline 4 & $\begin{array}{l}\text { Average of growth percentage on initial } \\
\text { variety and selected superior mutant } \\
\text { varieties }\end{array}$ & 77.00 & & & 91.60 & & \\
\hline 5 & Flowered time (d. a. p) & 55 & 38 & 39 & 40 & 41 & 40 \\
\hline 6 & Average flowered time (d. a. p) & 55 & & & 39.8 & & \\
\hline 7 & Leaf color & Green & Green & Green & Green & Green & Green \\
\hline 8 & Flower color & Yellow & Purple & Purple & Purple & Purple & Purple \\
\hline
\end{tabular}




\begin{tabular}{|c|c|c|c|c|c|c|c|}
\hline \multirow{2}{*}{ No } & \multirow{2}{*}{ Description } & \multirow{2}{*}{$\begin{array}{l}\text { Control } \\
\text { Sample }\end{array}$} & \multicolumn{5}{|c|}{ Selected Superior Mutant Varieties $\left(M_{n} V\right), n=1,2,3,4,5$} \\
\hline & & & 1 & 2 & 3 & 4 & 5 \\
\hline 9 & $\begin{array}{l}\text { Average of high plant on every sample } \\
(\mathrm{cm})\end{array}$ & 61.68 & 83.42 & 82.25 & 81.36 & 83.12 & 82.38 \\
\hline 10 & $\begin{array}{l}\text { Average of high plant on selected mutant } \\
\text { varieties }(\mathrm{cm})\end{array}$ & & & & 812,51 & & \\
\hline 11 & $\begin{array}{l}\text { Range of harvest time on every variety } \\
\text { (d. a. p) }\end{array}$ & $93-126$ & $80-82$ & $80-83$ & $80-82$ & $80-83$ & $80-83$ \\
\hline 12 & $\begin{array}{l}\text { Average range of harvest time on } \\
\text { selected mutant varieties }\end{array}$ & - & & & $80-82.6$ & & \\
\hline 13 & Total average of harvest time (d. a. p) & 109 & & & 81 & & \\
\hline 14 & $\begin{array}{l}\text { Range of pods number per tree on } \\
\text { every variety }\end{array}$ & $23-98$ & $126-257$ & $124-248$ & $125-246$ & $123-247$ & $124-249$ \\
\hline 15 & $\begin{array}{l}\text { Average of pods number per tree on } \\
\text { every variety }\end{array}$ & 61 & 192 & 186 & 186 & 185 & 187 \\
\hline 16 & $\begin{array}{l}\text { Total average of pods number per tree } \\
\text { on superior selected mutant variety }\end{array}$ & & & & 187.2 & & \\
\hline 17 & $\begin{array}{l}\text { Range of seeds number per pod on } \\
\text { every variety }\end{array}$ & $1-3$ & $2-5$ & $1-4$ & $1-3$ & $1-4$ & $1-3$ \\
\hline 18 & Skin color of dry pods & Brown & Brownish & Brownish & Brownish & Brownish & Dark brown \\
\hline 19 & Color seeds & Brownish & Yellowish & $\begin{array}{l}\text { White } \\
\text { yellow }\end{array}$ & Brown & Brown & Brownish \\
\hline 20 & $\begin{array}{l}\text { Average mass per } 1,000 \text { seeds }(\mathrm{kg}) \text { on } \\
\text { every variety }\end{array}$ & 0.18256 & 0.28124 & 0.27982 & 0.27687 & 0.27548 & 0.27615 \\
\hline 21 & Average mass per 1,000 seeds $(\mathrm{kg})$ & 0.18256 & & & 0.27771 & & \\
\hline 22 & $\begin{array}{l}\text { Adaptation to area with dry condition, } \\
\text { high salt, and calcium }\end{array}$ & No & Yes & Yes & Yes & Yes & Yes \\
\hline 23 & Tolerant to germ & No & Yes & Yes & Yes & Yes & Yes \\
\hline 24 & Adaptation to place altitude & No & Yes & Yes & Yes & Yes & Yes \\
\hline 25 & Protein content $(\%)$ & 12.40 & 13.82 & 13.78 & 13.76 & 13.74 & 13.75 \\
\hline 26 & $\begin{array}{l}\text { Production range on every variety } \\
(\mathrm{t} / \mathrm{ha})\end{array}$ & $1.96-2.61$ & $3.92-5.12$ & $3.87-4.95$ & $3.73-4.90$ & $3.76-4.92$ & $3.72-4.87$ \\
\hline 27 & $\begin{array}{l}\text { Total production range on superior } \\
\text { selected mutant varieties }(\mathrm{t} / \mathrm{ha})\end{array}$ & & & & $3,72-5,12$ & & \\
\hline 28 & $\begin{array}{l}\text { Average production on every variety } \\
(\mathrm{t} / \mathrm{ha})\end{array}$ & 2.40 & 4.53 & 4.52 & 4.46 & 4.47 & 4.44 \\
\hline 29 & $\begin{array}{l}\text { Total average production on superior } \\
\text { selected mutant variety }(\mathrm{t} / \mathrm{ha})\end{array}$ & & & & 4.48 & & \\
\hline \multirow[t]{2}{*}{30} & $\begin{array}{l}\text { Potential of maximum production } \\
(\mathrm{t} / \mathrm{ha})\end{array}$ & 2.61 & 5.12 & 4.95 & 4.90 & 4.92 & 4.87 \\
\hline & $\begin{array}{l}\text { Total average of increasing percentage } \\
\text { of production on superior selected } \\
\text { mutant variety (\%) }\end{array}$ & & & & 46.43 & & \\
\hline
\end{tabular}

Table 3. Production level at four provinces on control and treatment samples on final purifying as a result of multigamma irradiation of local soybean

\begin{tabular}{|c|c|c|c|c|c|c|c|}
\hline \multirow{2}{*}{ No } & \multirow{2}{*}{$\begin{array}{l}\text { Planting } \\
\text { Locations }\end{array}$} & \multirow{2}{*}{$\begin{array}{l}\text { Control Sample } \\
(\mathrm{t} / \mathrm{ha})\end{array}$} & \multicolumn{4}{|c|}{ Selected Mutant Varieties or Treatment Samples (t/ha) } & \multirow[b]{2}{*}{$M_{5} V(t / h a)$} \\
\hline & & & $M_{1} V(t / h a)$ & $M_{2} V(t / h a)$ & $M_{3} V(t / h a)$ & $M_{4} V(t / h a)$ & \\
\hline $\mathrm{I}$ & \multicolumn{7}{|c|}{ Province of South Sulawesi } \\
\hline 1 & Padang & 2.34 & 3.92 & 3.87 & 3.73 & 3.76 & 3.72 \\
\hline 2 & Tinoring & 2.45 & 4.38 & 4.35 & 4.31 & 4.29 & 4.32 \\
\hline II & \multicolumn{7}{|c|}{ Province of East Nusa Tenggara } \\
\hline 3 & Oesao & 2.58 & 4.86 & 4.84 & 4.81 & 4.87 & 4.85 \\
\hline 4 & Fukdale & 2.61 & 5.12 & 4.95 & 4.90 & 4.92 & 4.87 \\
\hline III & \multicolumn{7}{|c|}{ Province of Middle Sulawesi } \\
\hline 5 & Tentena & 1.96 & 4.16 & 4.32 & 4.26 & 4.28 & 4.26 \\
\hline IV & \multicolumn{7}{|c|}{ Province of West Sulawesi } \\
\hline 6 & Mamuju & 2.47 & 4.75 & 4.78 & 4.75 & 4.68 & 4.61 \\
\hline Aver & ge Production & 2.40 & 4.53 & 4.52 & 4.46 & 4.47 & 4.44 \\
\hline
\end{tabular}




\begin{tabular}{|c|c|c|c|c|c|c|c|}
\hline \multirow{2}{*}{ No } & Planting & \multirow{2}{*}{$\begin{array}{l}\text { Control Sample } \\
\text { (t/ha) }\end{array}$} & \multicolumn{4}{|c|}{ Selected Mutant Varieties or Treatment Samples (t/ha) } & \multirow[b]{2}{*}{$\mathbf{M}_{5} \mathrm{~V}(\mathrm{t} / \mathrm{ha})$} \\
\hline & Locations & & $M_{1} V(t / h a)$ & $\mathbf{M}_{2} \mathrm{~V}$ (t/ha) & $M_{3} V(t / h a)$ & $\mathbf{M}_{4} \mathbf{V}(\mathrm{t} / \mathrm{ha})$ & \\
\hline \multicolumn{2}{|c|}{$\begin{array}{l}\text { Total average production for } \\
\text { superior selected mutant } \\
\text { variety }\end{array}$} & - & & & 4.48 & & \\
\hline \multicolumn{2}{|c|}{$\begin{array}{l}\text { Increasing percentage of } \\
\text { production on every } \\
\text { superior selected mutant } \\
\text { varieties }\end{array}$} & - & $47.02 \%$ & $46.90 \%$ & $46.19 \%$ & $46.31 \%$ & $45.95 \%$ \\
\hline
\end{tabular}

\section{Statistical calculation}

Based on data in Table 1 and equation (1), growth percentage of control and treatment samples calculated as follow.

Growth percentage on control sample on final purifying at four provinces. The number of seeds observed on every variety about 50 seeds (sample randomly chosen). The average number of seeds is not growth on control sample about 11.5 seeds

$G P=\left(\frac{T_{A S}-A_{S G}}{T_{A S}}\right) \times 100 \%=\left(\frac{50-11.5}{50}\right) \times 100 \%=77.00 \%$.
Growth percentage on treatment sample on final purifying at four provinces (six planting locations) as follows: Average seed is not growth on treatment sample (selected superior mutant variety) about 4.2 seeds.

$G P=\left(\frac{T_{A S}-A_{S G}}{T_{A S}}\right) \times 100 \%=\left(\frac{50-4.2}{50}\right) \times 100 \%=91.60 \%$

Average production on every selected mutant variety of soybean on final purifying at four provinces (six planting locations).

Mutant-1 variety:

$$
\begin{aligned}
& A_{P T S M(1)}=\frac{P_{L 1}+P_{L 2}+P_{L 3}+P_{L 4}+P_{L 5}+P_{L 6}}{6}=\frac{3.92+4.38+4.86+5.12+4.16+4.75}{6} \\
& A_{P T S M(1)}=4.53 t / h a .
\end{aligned}
$$

Mutant-2 variety.

$$
\begin{aligned}
& A_{P T S M(2)}=\frac{P_{L 1}+P_{L 2}+P_{L 3}+P_{L 4}+P_{L 5}+P_{L 6}}{6}=\frac{3.87+4.35+4.84+4.95+4.32+4.78}{6} \\
& A_{P T S M(2)}=4.52 \mathrm{t} / \mathrm{ha} .
\end{aligned}
$$

Mutant-3 variety.

$$
\begin{aligned}
& A_{P T S M(3)}=\frac{P_{L 1}+P_{L 2}+P_{L 3}+P_{L 4}+P_{L 5}+P_{L 6}}{6}=\frac{3.73+4.31+4.81+4.90+4.26+4.75}{6} \\
& A_{P T S M(3)}=4.46 \mathrm{t} / \mathrm{ha} .
\end{aligned}
$$

Mutant-4 variety.

$$
\begin{aligned}
& A_{P T S M(4)}=\frac{P_{L 1}+P_{L 2}+P_{L 3}+P_{L 4}+P_{L 5}+P_{L 6}}{6}=\frac{3.76+4.29+4.87+4.92+4.28+4.68}{6} \\
& A_{P T S M(4)}=4.47 \mathrm{t} / \mathrm{ha} .
\end{aligned}
$$

Mutant-5 variety.

$$
\begin{aligned}
& A_{P T S M(5)}=\frac{P_{L 1}+P_{L 2}+P_{L 3}+P_{L 4}+P_{L 5}+P_{L 6}}{6}=\frac{3.72+4.32+4.85+4.87+4.26+4.61}{6} \\
& A_{P T S M(5)}=4.44 \mathrm{t} / \mathrm{ha} .
\end{aligned}
$$

Average production of initial variety at six planting locations (four provinces) on final purifying.

$$
\begin{aligned}
& A_{P C I V}=\frac{P_{L 1}+P_{L 2}+P_{L 3}+P_{L 4}+P_{L 5}+P_{L 6}}{6}=\frac{2.34+2.45+2.58+2.61+1.96+2.47}{6} \\
& A_{P C I V}=2.40 \mathrm{t} / \mathrm{ha} .
\end{aligned}
$$

Total average production of five variations of selected mutant varieties (treatment samples). 


$$
\begin{aligned}
& A_{P T S M(t)}=\frac{A_{P T S M(1)}+A_{P T S M(2)}+A_{P T S M(3)}+A_{P T S M(4)}+A_{P T S M(5)}}{5} \\
& A_{P T S M(t)}=\frac{4.53+4.52+4.46+4.47+4.40}{5}=4.48 t / h a .
\end{aligned}
$$

Increasing percentage of production on every selected superior mutant variety on final purifying:

Mutant-1 variety $\left(M_{1} V\right):_{P P S S M(1)}=\left(\frac{A_{P T S M(1)}-A_{P C I V}}{A_{P T S M(1)}}\right) \times 100 \%=\frac{4.53-2.40}{4.53} \times 100 \%=46.80 \%$.

Mutant-2 variety $\left(\left(M_{2} V\right):_{I_{P P S S M(2)}}=\left(\frac{A_{P T S M(2)}-A_{P C I V}}{A_{P T S M(2)}}\right)=\frac{4.52-2.40}{4.52} \times 100 \%=46.90 \%\right.$.

Mutant-3 variety $\left(M_{3} V\right): I_{P P S S M(3)}=\left(\frac{A_{P T S M(3)}-A_{P C I V}}{A_{P T S M(3)}}\right) \times 100 \%=\frac{4.46-2.40}{4.46} \times 100 \%=46.19 \%$.

Mutant-4 variety $\left(M_{4} V\right):_{I_{P P S S M(4)}}=\left(\frac{A_{P T S M(4)}-A_{P C I V}}{A_{P T S M(4)}}\right) \times 100 \%=\frac{4.47-2.40}{4.47} \times 100 \%=46.31 \%$.

Mutant-5 variety $\left(M_{5} V\right):_{I_{P P S S M(5)}}=\left(\frac{A_{P T S M(5)}-A_{P C I V}}{A_{P T S M(5)}}\right) \times 100 \%=\frac{4.44-2.40}{4.44} \times 100 \%=45.95 \%$.

Total average percentage of production of selected mutant variety at four provinces (six planting locations) on final purifying of soybean.

$$
\begin{aligned}
& A_{P T P S M V}=\left(\frac{I_{P P S S M(1)}+I_{P P S S M(2)}+I_{P P S S M(3)}+I_{P P S S M(4)}+I_{P P S S M(5)}}{5}\right) \\
& A_{P T P S M V}=\left(\frac{46.80+46.90+46.19+46.31+45.95}{5}\right)=46.43 \% \text {. }
\end{aligned}
$$

\section{Discussion}

\section{Growth Time, Growth Percentage, Flowered Time, and Adapted to Altitude}

Based on results of observation, measurement, and calculation, selected mutant varieties on final purifying have average growth time about 4 days after planting, while initial variety about 8 days after planting. This case caused by stimulation of irradiation from multi-gamma sources on selected mutant varieties. Average of growth percentage on final purifying for selected mutant varieties about $91.60 \%$, while initial variety about $77.00 \%$.

Average of flowered age on selected mutant varieties on final purifying is 39.8 days after planting, while initial variety 55.0 days after planting. That is show that selected mutant varieties as a result of development with using multi-gamma irradiation method on final purifying grow and flowered faster than initial variety.

Figure 2 up to Figure 6 clearly show that selected mutant varieties of soybean which planted at four Provinces on final purifying are fertile grow. That is shows that selected mutant varieties of soybean as a result of multi-gamma irradiation, carefully selection, and purifying can be adapted to area on altitude $>600$ meters from sea level and dry condition, and can be adapted to temperature $<23^{\circ} \mathrm{C}$. According to theory, soybean well grows in the area with altitude $<600$ meters from sea level (Atman, 2006) and temperature ranges about (23 up to $25^{\circ}$ ) C (Irwan, 2006).

\section{Number of Pods, Range of Seeds Number, and Mass per 1,000 seeds}

Selected mutant varieties on final purifying have average number of pods revolved between (185 up to 192) pods per tree with total average of pods per tree about 187 pods. Number of pods on initial variety about (23 up to 98) pods per tree with average of pods number per tree about 61 pods. This case can be proposed that number of pods per tree on selected mutant varieties and initial variety is significantly different.

Range of seeds number per pod on selected mutant varieties is similarly that is 1 seed per pod up to 5 seeds per pod, while initial variety 1 seed per pod up to 3 seeds per pod. Total average of mass on every group of 1,000 seeds on selected mutant varieties about 0.27771 $\mathrm{kg}$, while initial variety about $0.18256 \mathrm{~kg}$. That case shows that average mass per 1,000 seeds on selected mutant varieties is higher than mass per 1,000 seeds on initial variety on final purifying.

\section{Soybean age}

Based on observation results were clearly shown that soybean seeds which irradiated by multi-gamma sources, carefully selection, and purifying growth faster than initial variety. Average growth time of superior selected mutant varieties about 4 days after planting 
with average of growth percentage about $91.60 \%$, while initial variety 8 days after planting with growth percentage about $77.00 \%$.

Average of flowered time on superior selected mutant varieties on final purifying about 39.8 days after planting while initial variety about 55 days after planting. Range of harvest time on superior selected mutant varieties 80.0 days after planting up to 82.6 days after planting with average of harvest time about 81 days after planting, while initial variety about 93 days after planting up to 126 days after planting with average of harvest time about 109 days after planting.

That result shows that superior selected mutant varieties of soybean as a result of multi-gamma irradiation, carefully selections, and purifying flowered faster than initial variety.

\section{Tolerant to Germ}

Figure 2 up to Figure 6 show that growth of superior selected mutant varieties of soybean on final purifying were very fertile with leaves clearly visible were pure. Figure 7 up to Figure 11 also clearly show that fruit of superior selected mutant varieties clearly visible are not attacked of germ. That case shows that superior selected mutant varieties can be tolerant to germ.

\section{Average of Increasing Production and Protein Content}

Calculation results show that total average production of superior selected mutant varieties on final purifying were higher than initial variety. Productions range on all superior selected mutant varieties and initial variety respectively 3.72-5.12 t/ha and 1.96-2.61 t/ha. Range of average production of superior selected mutant varieties at six planting locations in 4 provinces about 4.44-4.53 $\mathrm{t} / \mathrm{ha}$. Total average production of superior selected mutant varieties about $4.48 \mathrm{t} / \mathrm{ha}$, while initial variety about $2.40 \mathrm{t} / \mathrm{ha}$ with total average of increasing percentage of production $46.43 \%$. That results show that production of superior selected mutant varieties was higher than production of initial variety. Protein content of initial and superior selected mutant varieties respectively (service analysis) about, 12.40\%, 13.82 (mutant-), 13.78\% (mutant-2), 13.76\% (mutant-3), $13.74 \%$ (mutant- 4 ), and $13.75 \%$ (mutant-5)

\section{Conclusion}

Based on explanations upon can be proposed conclusions as follow. Continuous development of soybean on final purifying obtained 5 variations of superior selected mutant varieties with average production on every variety was relatively same. Superior characteristics on every variety of superior selected mutant varieties a large part was similarly like as flowered time, growth percentage, harvest time, and so on. A few different likes as seeds measure, pods number per tree, plant high, seeds color, and so on. Total average production of superior selected mutant varieties on final purifying was higher than production of initial variety. Total average production of superior selected mutant varieties on final purifying about $4.48 \mathrm{t} /$ ha with total average of production increasing percentage about $46.43 \%$ while initial variety $2.40 \mathrm{t} / \mathrm{ha}$.

\section{Acknowledgments}

This research or study was supported and funded by Decentralization research, Directorate of Research and Public Service, Department of Education and Culture of Indonesia Government (KEMENRISTEK DIKTI), March, 2014 up to March, 2017 [grant number 21/UN15.19.1.2/ SP2H/PL/ 2014 (the first and the second year), number 142/UN15.19.1/LT/2016 (the third year), and number 199/UN15.19/LT/2017 (the fourth year)]. Especial thanks to Government of Indonesia has been funded of this research and also especial thanks to all colleagues in English Training Centre, whom have helped me in technical writing of this manuscrip.

\section{References}

Aimon., H., Satrianto., \& Alpon. (2014). Consumption Prospect and Import of Soybean in Indonesia, 2015 - 2020. Journal of Economics Study, III(5). Country University Padang.

Arsyad, D.M \& Syam, M. (1998). Soybean Increasing Source of Production and Cultivation Technique, Puslitbangtan.

Atman. (2014). Soybean Production: Strategy Increase production of Soybean through PTT. Yogyakarta.

Azis. (2014). Factors Influence value of Soybean in Indonesia, Jakarta. Scientific Bulletin Trade Litbang, $18(1)$.

Azzamy. (2015). Disease and Pest of Soybean.

Atman. (2006). Cultivation of Soybean at wet rice field, BPTP West Sumatera.

Baliardi, Y., \& Saleh, N. (1989). The main Viruses in production Center of Soybean in East Java. Proceeding on National Congres $X$ and Scientific Seminar PFA, Dempasar.

Darussalam, M. (1989). Radiation and Radioisotopes, the principle Using in Biology, Medicine, and Agriculture, Tarsito. Bandung.

Hasan, N., Erma Suryan., \& Hendrawan, R. (2015). Analysis of Soybean Production and Demand to Develop Strategic Policy of Food Self. Procedia Computer Science 72; 605 - 612. 
Indrawan, S. (2009). The New Superior Variety of Soybean as a Result of Breeding with Radiation Method. Journal Science and Nuclear Technology, 1(6), 12-38. Retrieved from http:// sony42.wordpress.com/2009/02/26/

Irwan, A. W. (2006). Cultivation of Soybean, Jatinangor.

Krisnawati., Ayda., \& Adie, M.M. (2015). Selections of Soybean Genotypes by Seed Size and its Prospects for Industrial Raw Material in Indonesia. Procedia Food Science, 3, pp. 355-363.

Mansyuriadi. (2015). Analysis of Production Increasing of Soybean in Indonesia, Agro-economics Journal, 17(1). 27-36.

Marwoto., Hardaningsih, S., \& Taufiq, A. (2017). Disease and Pest of Soybean. Identification and Restraint, Research Center and Plant Food Development, Organization of Research and Agricultural Development.

McFarlane, I., \& O'Connor. (2014). World soybean trade: growth and sustainability. Modern Economy, Vol 5(5). 580-588.

Nancy, O., Hisjam, M., \& Sutopo, W. (2018). The dynamic simulation model of soybean in Central Java to support food self sufficiency: A supply chain perspective. AIP Conference Proceedings 1931, $030015 \quad$ (2018); https://doi.org/10.1063/1.5024074

Okada, T., Tengkono, W., \& Djuwarso, T. (1988). An Outline of Soybean Pest in Indonesia in Faunistic Aspects, Seminar Balittan Bogor.

Pasangaka, B., \& Jaelani, A.K. (2010). The Breeding of Local Corn by Multi-gamma Radiation Method (Nuclear) in West Timor East Nusa Tenggara Indonesia, Journal of Technoscientia, 3(1), 11-21.

Pasangka, B., \& Refli. (2013a). Developing of Local Peanut Based on Multiculture That Tolerant to Abiotic and Biotic Conditions with Multigamma Radiation Method (Nuclear). Journal of Agricultural Science; 5(5). 15-25. Published by Canadian Center of Science and Education, URL: http://dx.doi.org/10.5539/jas.05n5p15, .

Pasangka, B., \& Refli. (2013b). The Application of Multigamma Radiation as a Physical Mutagen for Breeding of Local Soybean, Journal of Agricultural Science; 5(6). 201-210. Published by Canadian Center of Science and Education,

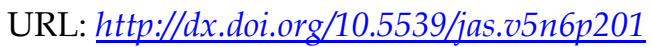

Pasangka, B., \& Refli. (2016). Development and Cultivation of Local Kidney Bean (Phaseolus Vulgaris L) Through Breeding to use Multi gamma Irradiation Technique (Nuclear), American Journal of Agricultural and Biological Sciences, Science Publications, URL: https://thescipub.com., DOI: 10.3844/ajabssp.2016.117.127, pp. 117-127.
Pasangka, B. (2018). Cultivation of Soybean as a Result of Genetic Mutation with Nuclear Technique (Multigamma Irradiation) Final Revision Edition, Publishing UNDANA Press and Nusa Indah.

Pasangka, B. (2019). Cultivation of Vigna Radiata L as a Result of Genetic Mutation with Nuclear Technique, Fist Edition, Publishing UNDANA Press and Nusa Indah.

Saktyanu, K.D. (2014). Policy Analysis for Indonesia's Rice, Maize and Soybean Development in Dealing with ASEAN Free Trade, Central of Economic Social, Journal of Economic Study, 8(5).

Statistics Center Organization. (2014). Statistical Year Book of Indonesia. Jakarta.

Zakaria A. K., Wahyuning, K.S., \& Kustiari, R. (2018). The Analysis of Competitiveness of the Soybean Commodity According to Agro the Ecosystem: The Case in Three Provinces in Indonesia, Analysis Center of Economic Social and Agriculture Policy. Jurnal Agro Ekonomi, 28(1), 2137. 